\title{
Application Domain Accounting for Roaming Services
}

\author{
Paolo Bellavista, Antonio Corradi, Silvia Vecchi \\ Dip. Elettronica, Informatica e Sistemistica - University of Bologna \\ \{pbellavista, acorradi,svecchi\}@deis.unibo.it
}

\begin{abstract}
Wireless portable devices and ubiquitous service access points are enabling pervasive scenarios where a plethora of different client terminals expect to access services while roaming from a network locality to another one and between different local service providers. Within this usage perspective, there is the need for extending accounting functions to include also the possibility to charge clients for application-level resource consumption, i.e., application domain accounting. The paper claims that application domain accounting of roaming services requires extending the fixed Internet infrastructure, where and when needed at service provisioning time, with mobility-enabled monitoring, processing, pricing and charging functions. We propose an accounting middleware solution capable of evolving dynamically in dependence of the exhibited mobility patterns of Wi-Fi client devices. The presented solution is based on the Mobile Agent (MA) implementation technology, on portable mechanisms for quality monitoring, and on portable mechanisms for achieving the visibility of IEEE $802.11 \mathrm{~b}$ client roaming at the middleware level to automatically trigger the migration of the needed middleware components.
\end{abstract}

\section{Introduction}

The spectrum of Internet-based services offered to final users is continuously enlarging, by considering service provisioning to clients connected to either fixed networks or mobile ones. In addition, the organization requirements connected to the increasing capabilities of wireless access devices are deeply modifying the service provisioning process. On the one hand, $3 \mathrm{G}$ mobile networks should support services that present the same richness and variety of the ones provided to fixed environments. On the other hand, the availability of very heterogeneous portable devices is likely to change some aspects of service provisioning, for instance by forcing to consider service delivery anywhere and anytime with an ad-hoc dynamic adaptation to the properties of the current access terminals.

This new perspective calls for services that can be provided to users while they roam in a globally distributed network with their wireless portable devices connected to local/global service providers (SPs) [1, 2]. Client roaming is a widely common telecom service functionality, which is supported by most cellular phone operators; we claim that a similar concept of client roaming needs to be extended to the emerging novel classes of Internet-based mobile services. Similarly as a phone user roams and seamlessly passes between possibly different network operators, roaming customers of Internetbased mobile services should subscribe to a specific service and should have the possibility to connect to the most suitable SP available, e.g., depending on the client location. Let us consider a city guide service allowing clients to get information about shops, restaurants, and cinemas. A user should have the possibility to subscribe to one single SP providing the city guide, and to access the service even while roaming in other cities where other SPs offer a similar service. In addition, tourist information should be dynamically adapted to fit the specific access device visualization capabilities and should be filtered according to the specified/exhibited user preferences. In the following, we indicate such novel class of services as Roaming Services.

Roaming service scenarios raise several challenging issues to investigate and solve. The paper specifically explores the accounting area, i.e., how to collect and manage the resource usage information in roaming service provisioning for charging and billing purposes. The resources to be considered in the accounting process are very heterogeneous in nature and in their possible implementation, spanning from used network bandwidth to consumed processor time and memory, from the size of actually received data to the exploited functions for dynamic adaptation. In the provisioning of roaming services, we can distinguish between the accounting part related to pure network connectivity and the accounting part associated with application-level service aspects. For instance, in the city guide service, the network operator can be interested in accounting roaming users for the time they exploit the network connectivity, but also the guide SPs can require to account them for the 
amount (number of pages), the quality (textual/multimedia presentation), the access mode (with/without adds), and the dynamic adaptation (downscale of image resolution or format conversion) of the service contents.

This suggests a separation between network domain and application domain, as defined in the UMTS specifications [3]. In general, the network domain defines the management aspects related to the access to the network infrastructure and to the transport-related issues, such as traffic control. The application domain considers the management properties of the service per se and possible additional requirements stemming from the specific contents provided (text, audio stream, video stream, ...). The two domains motivate also different approaches and solutions for accounting management. Network domain accounting generally adopts time-based pricing, possibly differentiating the cost per time unit depending on the time of the day. This simple model is typically not applicable to the more complex service aspects belonging to the application domain, where, for instance, there is the need to consider the actually received level of Quality of Service (QoS) and also the dynamic tailoring effort to downscale the service contents to the specific capabilities of the client access device.

In roaming services, similarly as in cellular phone voice ones, the network domain cost management can be organized as follows. It is the Foreign SP (FSP) that can meter the network usage of roaming clients, and can ask the responsible Home SP (HSP) to pay for it by sending usage reports either directly to HSP or to an agreed third party. This accounting strategy is based on the assumption that FSPs are capable of metering the consumption of roaming users, e.g., by registering the seconds spent in a phone call. This solution model, however, is viable only for simple and largely recognized metering operations, usually carried out via standard lower-level accounting mechanisms. Considering the application domain significantly changes the perspective because application domain accounting management usually requires to have visibility of very heterogeneous application-specific service properties and possibly to enforce very different charging policies for different SPs. In addition, it is often necessary to take into account personalized pricing policies resulting from conditions that are negotiated between single users and their SPs.

The paper presents the design and implementation of a novel accounting solution, called roaming accounting, where a HSP can dynamically delegate FSPs to account its users while they roam in different network localities. The proposed accounting middleware implements this form of delegation by shipping the needed metering and charging logic to the currently involved FSPs at service provisioning time. This approach permits to apply com- plex and possibly differentiated billing policies to different classes of users even while roaming. Our solution is mobile in the sense that accounting middleware components can migrate where needed depending on client mobility patterns by exploiting a Mobile Agent (MA)based implementation. The roaming accounting system is built on top of the Secure and Open MA (SOMA) platform and exploits a layer of portable middleware components for application-level monitoring and charging [4] [5].

The rest of the paper is organized as follows. Section 2 gives a brief overview of some existing roaming scenarios, i.e., mobile telephony, dial-up Internet and wireless Internet. Section 3 sketches the billing schemes adopted in the previously presented scenarios, by pointing out the motivations for considering also application domain aspects and the corresponding requirements for extending state-of-the-art accounting solutions. Then, Section 4 presents the architecture of our roaming accounting system; some implementation technology insights about the proposed solution are given in Section 5. Conclusive remarks and directions of future work end the paper.

\section{Roaming Scenarios}

Roaming is usually defined as the ability of clients to access services from different SPs while having an explicit subscription with only one of them [2]. Roaming is an established feature of mobile telephony and also, although to a lesser extent, of dial-up and wireless Internet access scenarios. To better understand the motivations of our roaming accounting proposal, let us briefly outline here these three existing roaming scenarios, respectively mobile telephony, dial-up Internet and wireless Internet.

Roaming in mobile telephony relates to significantly different functions in $2^{\text {nd }}$ generation and in $3^{\text {rd }}$ generation mobile networks. Services supported in the Global System for Mobile telecommunications (GSM) are mainly voice calls and Short Message Service (SMS); these GSM services are provided directly by network operators, and consequently the roaming process only involves the network operators. Roaming support is part of the GSM standard, and GSM operators also cooperate to achieve agreements on the business/technology aspects of roaming not addressed by the standard specification. The roaming scenario in Universal Mobile Telecommunications System (UMTS) is quite different. UMTS encourages the enrichment and extension of traditional voice services via partnering of network operators and application/content providers. UMTS users can roam and pass from one network operator to another one, as in GSM networks, but they can also roam between application/content SPs, independently of the 
specific network locality where they first registered. The roaming issues of service provisioning over UMTS are currently subject of several research work [1].

Subscribers of traditional dial-up Internet services generally use a fixed Internet SP (ISP) and a fixed telephone number to enable their access, independently of their current location. This is more expensive and less effective, in terms of both connection time and transmission quality, than connecting via a locally equivalent ISP. In dial-up Internet, roaming refers to the ability of accessing the Internet via a dial-up modem connection to an ISP chosen among the ones close to the current user locality, with no need for an explicit previous subscription with that ISP. In current solutions for dial-up Internet roaming, client applications help users in finding the phone numbers of close ISPs with roaming arrangements/agreements (direct or via roaming brokers) with their HSPs.

The increasing popularity of Wireless LAN (WLAN) is creating a new market of Wireless ISPs (WISPs) that provide users with the accessibility to either the Internet or a corporate intranet in specially equipped areas, such as hotel rooms, airports or trade fair halls [2]. The number of these wireless access points, usually identified as hot spots, is constantly growing. WISPs are continuously extending their coverage, thus making more and more crucial to set inter-WISP roaming agreements. Roaming in this scenario relates to the ability to access WLANs of different WISPs with no need for an explicit previous subscription with those WISPs. The provisioning of Internet services with WLAN accessibility is still in its infancy, and several aspects of roaming service management are at the state-of-the-art. However, WLAN-enabled scenarios for $\mathrm{m}$-commerce and location-dependent provisioning are attracting many network operators and SPs, thus making crucial to investigate and develop novel suitable middleware solutions to support roaming services.

\section{Accounting Issues in Roaming Scenarios}

A crucial factor to address in roaming service provisioning is the accounting management, i.e., how to measure, control, collect and process the distributed information about resource usage for charging and billing purposes. The three roaming scenarios presented above tend to adopt different accounting models and strategies; let us first examine them to better understand the motivations for novel and more flexible middleware solutions for application domain roaming accounting.

In GSM roaming, billing solutions are based on the Call Detail Record (CDR) generated in the visited network. The CDR is generally transferred to a clearinghouse, i.e., a company in charge of ruling and certifying clearing and financial settlement between service providers [2], and then forwarded to the home operator of the customer. To support CDR portability, the GSM association has specified the Transferred Account Procedure (TAP), a set of standard formats and procedures for the exchange of roaming-related billing information between network operators and clearinghouses [2].

In dial-up Internet, roaming ISPs typically do not have direct mutual roaming agreements, but only an agreement with a roaming broker. The roaming broker negotiates a wholesale rate with each of the served ISPs: it is the roaming broker that will pay the visited ISP for the time of network connectivity used by its roaming clients and that will charge the home ISP for that usage.

While roaming is not considered today a highpriority competitive advantage for dial-up ISPs, it is already recognized that it represents a key feature for WISPs, even if accounting models, strategies and solutions are not yet well established in the wireless access scenario. However, the main guideline emerging is to adopt similar business and billing models as in GSM roaming, with a direct arrangement between SPs.

These briefly sketched approaches represent the state of the art in roaming accounting solutions. They clearly show how the current accounting focus is on billing models and payment rules, as with the implicit assumption that users should be charged exclusively on the basis of their access time to services. Only within this simplified perspective it is possible to assume that FSPs can account roaming users without having further knowledge about the HSP-specific accounting strategies. For instance, in this perspective the only monitoring operation needed is to measure the time interval of users' access to the provided services. This approach is applicable in network domain accounting and is suitable for services where the usage of the network infrastructure is the fundamental accounting aspect, such as in telephony services.

When dealing with advanced Internet services for wireless portable devices such as the city guide one, there is the need for application domain accounting solutions where users can be charged also for the accessed service contents, the actually received QoS levels, and the needed dynamic adaptations of content formats. To this purpose, it is necessary to enable the metering of several different resources at different levels of abstraction, spanning from the bandwidth used in the access network locality to the consumed processor/memory on the content adaptation nodes. In general, it is crucial to consider also complex and application-level metering indicators that may be specific for the provided services, the used access devices, and the Service Level Agreements (SLAs) between the clients and their HSPs.

In application domain accounting, it is no more practical to assume that FSPs statically and a-priori know which types of resource consumptions the inco- 
ming roaming users must be charged for. Any SP can host users from several different HSPs, each one with a different provisioning and faring policy. To achieve roaming accounting, FSPs should know not only which pricing strategies to apply and when, but also which resource consumption indicators to meter and how.

\section{Roaming Accounting Architecture}

We claim that application domain roaming accounting needs novel, dynamic and flexible middleware solutions to enable very differentiated billing schemes at service provisioning time and to assist the FSP developers in the treatment of all the accounting-related implementation complexity. We propose a middleware, indicated as roaming accounting in the following, where any HSP can delegate FSPs to account its users while they roam in the network, by shipping both metering and charging logic locally to the users, when and where needed. The possibility to move metering/charging logic at runtime permits to achieve several advantages. First, an FSP does not need to know statically the accounting logic applicable to any potential roaming user visiting its network locality; the FSP does only offer its computational resources to carry out the accounting operations performed by the HSP shipped logic. Second, metering/charging logic mobility may avoid the transfer of large amounts of raw monitoring information between SPs or between SPs and clearing houses; this transfer is generally needed to process the monitoring data for billing purposes locally either to the involved HSP or to the interested clearing house.

Along the above guidelines, we have designed and implemented an accounting middleware solution for roaming environments where clients exploit Wi-Fi network connectivity to access Internet services provided over the fixed network infrastructure. We have chosen the IEEE802.11-enabled WISP scenario because of the definite lack of well-established accounting solutions in this context and the growing interest recently shown by the industry and tourism communities for Wi-Fi connectivity. However, our roaming accounting approach is of general applicability, and can be implemented and deployed over any of the previously presented roaming scenarios.

Service usage metering and charging are the basic functions required in any accounting management solution, also when considering traditional accounting over the fixed Internet infrastructure. Metering components usually work at the network domain level and are available at any host providing network accessibility. Their behavior is driven by the local metering logic, which is statically installed and depends on the accounting strategy settled by the local SP, typically the FSPs in roaming scenarios. On the contrary, charging tasks are usually performed only in special billing hosts, where the metering data are collected and processed (typically off-line) accordingly to the local billing policies, i.e., the billing policies of the user HSP.

On the contrary, in our roaming accounting middleware, metering components operate at both the network domain level and the application domain one, and are available in any host providing network accessibility and/or service contents. They can perform userspecific programmable tasks, which are expressed by the metering logic shipped by the user-responsible HSP. In addition, there are no centralized billing hosts: charging tasks are completely distributed both in space and in time. Charging tasks are spatially decentralized because they are performed directly in the host where the service is accessed; moreover, they run at service provision time, thus permitting also the enforcement of dynamic pricing strategies.

Our metering and charging functions are implemented as mobile middleware components capable of migrating during service provisioning, to reach the access locality where the roaming users are accounted locally. The roaming accounting middleware exploits the Mobile Agent (MA) implementation technology and, in particular, is built on top of SOMA. SOMA is a Java-based general-purpose platform for the design, development and deployment of MA services in global, open and untrusted environments [4].

The architecture of our roaming accounting middleware consists of three main services: the Metering Service, the Charging Service, and the Location Service (see Figure 1).

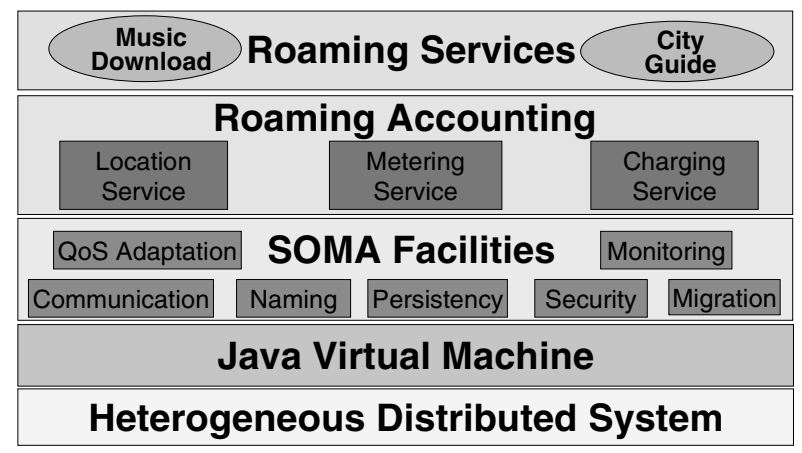

Figure 1. The architecture of the roaming accounting middleware.

The Metering Service is implemented in terms of Metering Mobile Agents (MMAs) that encapsulate the metering logic to apply to specific classes of users and that dynamically migrate to the wireless access localities where the associated accounted users roam during service provisioning. Once local to their roaming clients to account, MMAs exploit the local monitoring mecha- 
nisms available in any SOMA-enabled host to inspect and extract data about user-specific local resource engagement. In fact, each SOMA execution environment is equipped with a highly configurable and dynamically extensible monitoring module that can trace the usage of different types of resources, at both the system level and the application one. MMAs locally configure the monitoring modules only to trace the resource consumptions that are specified in their metering logic and needed for their user-specific charging; this permits to effectively tune the intrusiveness of on-line monitoring and to significantly reduce its overhead [5].

The Charging Service is realized by Charging Mobile Agents (CMAs) that encapsulate the charging logic to apply in order to produce the final billing reports for the users accordingly to their SLAs. CMAs move to the network localities visited by the accounted roaming users at service provision time, locally process the accounting relevant data which have been metered and locally stored by MMAs, and finally compile the billing reports in a completely decentralized way, either just at the end of the user service session or off-line at specified time intervals.

To enable the automatic shipping of MMAs and CMAs to the needed FSPs, our roaming accounting middleware must have full awareness of the IEEE802.11 cells where users are currently located and accessing the services to be accounted for. To this purpose, roaming accounting includes a Location Service which permits to overcome the typical location transparency of most implementations of Wi-Fi-compliant WLANs. In particular, as detailed in the next section, our location service exploits portable mechanisms to achieve the on-line visibility of the position of wireless client devices and makes the location information easily accessible to the other middleware services.

\section{Roaming Accounting Implementation Insights}

The roaming accounting middleware models any kind of interconnected system, from simple Intranet LANs to the Internet, in terms of locality abstractions, i.e., places, default places, and domains. Any network node hosts one place for the execution of MMAs and CMAs; several places can be grouped into domain abstractions, each one usually corresponding to one network locality. For instance, one domain can typically model the group of fixed hosts interconnected in one LAN and the wireless devices that are currently accessing it via local service access points. In each domain, a default place is in charge of inter-domain routing functionality (see Figure 2).

In the following, we give some design and implementation insights on how our middleware realizes the location, metering and charging services in Wi-Fienabled domains. In particular, the implementation of the location service and of the metering one requires addressing some technical challenges at the state-of-theart, especially when choosing to work at the application level over standard connectivity and programming solutions. Section 5.1 is devoted to clarify how to achieve cell-location visibility independently of the vendor implementation of the IEEE802.11 wireless clients, while Section 5.2 illustrates how to obtain on-line monitoring indicators even working on top of the Java Virtual Machine (JVM) and how MMAs and CMAs perform the accounting on the basis of these indicators.

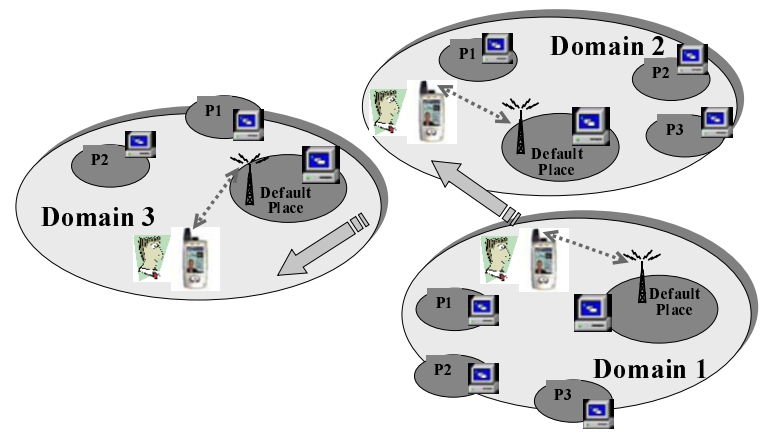

Figure 2. The roaming accounting locality abstractions.

\subsection{The Location Service}

IEEE802.11-compliant wireless solutions tend to hide service developers from all the complexity and details of mobility management. Application-level mobility transparency is desirable from the user point of view, but could limit both service designers interested in implementing location-aware services and systems/service administrators willing to consider management aspects related to client mobility, as in the case of roaming accounting. The location service is in charge of achieving full on-line visibility of the client celllocation, thus enabling the possibility for our roaming accounting middleware to trigger the migration of MMAs and CMAs in the localities where the users are currently accessing the accounted services.

Any domain hosts a location server running in the domain default place. The location server directly collects information about the clients currently connected to the fixed network via its local service access points. To obtain this information, the location server exploits the standard Simple Network Management Protocol (SNMP) [6]: in particular, the location server receives roaming-specific SNMP traps from the SNMP agents activated on any wireless service access point in its locality. SNMP traps are triggered by any new client terminal entrance/exit in/from the served Base Service Set 
(BSS), i.e., the locally served IEEE802.11 [7]. This solution is portable on all the most spread IEEE802.11 implementations; in fact, all major vendors have decided to support standard SNMP agents in their Wi-Fi service access points.

The notification of the BSS change of a client automatically triggers the generation and posting of a migration command to all the client-responsible MMAs, which receive the command independently of their current location by exploiting the SOMA mobility-enabled naming and messaging service [4]. On the contrary, CMAs follow automatically the user roaming when the user-specific charging operations should be executed online during service provisioning (see the example in the following section) or, more usually, simply track the user roaming in order to repeat the same migration path, off-line and later, when the service administrators decide to launch the charging and billing processes.

\subsection{The Metering and Charging Services}

As already sketched in Section 4, the metering service is built on top of monitoring modules that are available in any place and that locally inspect a wide set of system/application level indicators about resource consumption. At the system level, any monitoring module can get information about the processes working on local resources and about their usage of the communication infrastructure. For any process, it can report the process identifier and name, the CPU usage (time and percentage, of both the process and the composing threads) and the allocated memory (both physical and virtual). Network metering data include, for any process, the total number of sent/received UDP packets, of sent/received TCP segments, of TCP connections, and of TCP/UDP packets received with errors. At the application level, monitoring modules can collect information about all service components accessed from within the Java execution environment. For any active Java thread, they can detect any invocation of a dynamically definable set of methods and any object allocation/deallocation operation.

To overcome the transparency imposed by the JVM, the local monitoring module exploits some extensions of the Java technology: the JVM Profiler Interface (JVMPI) [8] and the Java Native Interface (JNI) [9]. In addition, it adopts the Java Management eXtensions (JMX) [10] to observe the state of both application-level Java components (modeled as JMX-manageable resources) and legacy monitoring systems, such as SNMPbased ones [6], as depicted in Figure 3. JVMPI makes possible to instrument dynamically the JVM for debugging and monitoring purposes, and the monitoring module exploits it to collect, filter and analyze applicationlevel events produced by Java-based components not explicitly designed in compliance with the JMX model. At the kernel level, the monitoring module collects system-dependent data by exploiting JMX to interface with SNMP agents exporting standard Management Information Bases (MIBs). To enable also the monitoring of hosts without any SNMP agent in execution, the monitoring module exploits JNI to integrate with platformdependent metering mechanisms, which we have currently implemented for the Windows NT, Solaris, and Linux platforms. Additional details about the implementation and the performance results of the adopted monitoring mechanisms are available in [5].

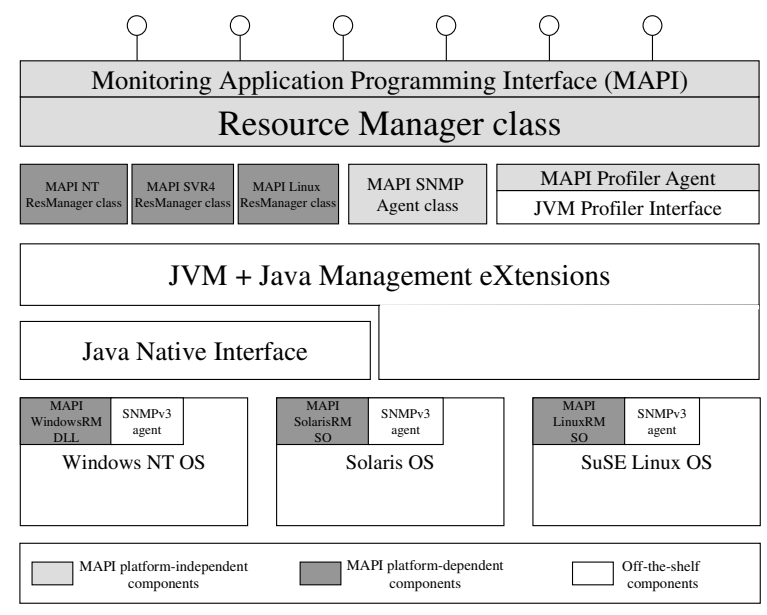

Figure 3. The architecture of the monitoring module

The monitoring module launches a new metering task anytime a user starts accessing services residing in its place, and forces the task termination at the end of the user service session. Metering tasks are dynamically tuned by the MMAs, which follow the roaming clients and choose both the type of resources to monitor and the accuracy/frequency of the monitoring probing. This permits to enforce user/service-dependent metering logic, by only measuring the parameters that are relevant for the billing policies agreed between the users and their SPs.

The user-specific produced metering data are locally processed by the CMAs to quantify concisely the resource consumption according to the user-specific policies enforced for service faring. For instance, networkrelated information can be used to determine the overall network traffic generated by a user in a service session. The CPU usage and the memory allocation on a place can represent a rough estimate of the computational costs associated with some service downscaling operations, e.g., when converting an XML-based Web page into the reduced corresponding Wireless Markup Language (WML) one. In addition, monitoring the invoca- 
tion of specific server methods permits to sense the access to specific service content.

In the default CMA implementation of the roaming accounting middleware, one CMA follows a roaming user while processing and collecting her resource usage information in the different visited localities. The MA property of maintaining the reached execution state between migrations allows CMAs to trace the history of her user's service usage and charging, by permitting also the enforcement of pricing strategies depending on previous service usage. For instance, let us consider a music download service where the enforced pricing policy specifies the normal possibility to download songs at a price/song modeled as $f(x)$, where $x$ is the actually received QoS level. Good clients downloading more than $y$ songs in the last month, however, should be charged with a minor price/song $g(x)$. To enforce such a billing policy, it is necessary to know, in any visited domain, the number of downloads already performed by the user, sometimes together with the corresponding QoS level received, for instance to establish if a pre-paid service quota has already been totally consumed.

\section{Conclusions and On-going Work}

The Internet is likely to offer a pervasive and ubiquitous environment for service provisioning and this requires extending the fixed network infrastructure, when and where needed, to support dynamic service adaptation to mobile users and portable devices, while they roam between different network localities, different WISPs and different content providers. In this context, effective accounting solutions should consider also the application domain resources actually consumed during service provisioning, in order to stimulate a fair resource sharing among users and to leverage the market of services with differentiated quality levels. The paper shows how highly dynamic support infrastructures exploiting mobile code programming paradigms are particularly suitable for the design and implementation of application domain accounting in roaming services.

The encouraging results achieved by the first implemented prototypes of application domain roaming accounting on top of SOMA (see http://lia.deis.unibo.it/Research/Accounting/) are stimulating further work, on the one hand, to facilitate the development of dynamically adaptive roaming services, and, on the other hand, to produce a complete pervasive accounting system.

We are working on extending our roaming service infrastructure to support the automatic reconnection of roaming users to new local SPs that offer service functions similar to the ones they subscribed to in their HSP. We are defining service classes including SPs with common characteristics and functionality, and we are working to describe those characteristics in a standard representation format suitable for open provisioning environments such as the Internet. Let us note that UMTS similarly tend to specify service capabilities and not services per se [8]. In particular, we are adopting the emerging Web Services Description Language for a uniform representation of service characteristics/interfaces and of the tailoring/adaptation functions that our roaming middleware makes available locally to the client [12].

In addition, we are integrating our roaming accounting system with security solutions for the establishment of trust relationships. In particular, we are adapting the SOMA security mechanisms to authorize/control the execution of incoming MAs to the specific case of accepting metering and charging code from already known HSPs. The establishment of trust relationships between different network localities may reduce the security costs, by permitting the flexible and dynamic choice of the most suitable security mechanisms and policies depending on the specific deployment scenario [13].

Finally, we are working on the adoption of portable information models to represent the roaming accounting data and of high-level declarative specifications to express the accounting policies to be enforced. After investigating and analyzing the related proposals at the stateof-the-art, we have chosen to adopt the charging extensions to the XML-based Master Internet Protocol Detail Record (IPDR) [14] as the portable format for the accounting data.

\section{Acknowledgments}

Work supported by the Italian Ministero dell'Istruzione, dell'Università e della Ricerca in the framework of the FIRB Project "Wide-scale Broadband Middleware for Network Distributed Services" and the CNR Project "Middleware Support for Mobile Ad-hoc Networks and their Application".

\section{References}

[1] S. Panagiotakis, E. Gazis, M. Koutsopoulou, N. Houssos, Z. Boufidis, A. Alonistoti, "Roaming Issues for Service Provisioning over $3^{\text {rd }}$ Generation Mobile Networks", Advanced Technologies, Applications and Market Strategies for $3 G$ (ATAMS`01) Conference, June 2001.

[2] G. Almgren, "Roaming Between Wireless ISPs", White Paper, Service Factory www.servicefactory.com.

[3] 3G TS 22.101: "3rd Generation Partnership Project; Technical Specification Group Services and Systems Aspects; Services Principles (3G TS 22.101 version 3.12.0)". 
[4] P. Bellavista, A. Corradi, C. Stefanelli, "Mobile Agent Middleware to Support Mobile Computing", IEEE Computer, Vol. 34, No. 3, Mar. 2001.

[5] P. Bellavista, A. Corradi, C. Stefanelli, "Java for On-line Distributed Monitoring of Heterogeneous Systems and Services", The Computer Journal, Oxford University Press, November 2002, pp 595-607.

[6] J. D. Case, et al., "Simple Network Management Protocol (RFC 1157)", DDN Network Information Center, SRI International, May 1990.

[7] M Rose, "A convention for Defining Traps for use with SNMP”, RFC 1215

[8] Sun Microsystems - Java Virtual Machine Profiler Interface (JVMPI),

http://java.sun.com/prducts/jdk/1.3/docs/guide/jvmpi/jvmpi.ht $\mathrm{ml}$.

[9] Sun Microsystems - Java Native Interface (JNI), http://java.sun.com/ products/jdk/1.3/docs/guide/jni.html.
[10] Sun Microsystems - Java Management Extensions (JMX), http://java.sun.com/products/JavaManagement/doc.html.

[11] 3G TS 22.105: "3rd Generation Partnership Project; Technical Specification Group Services and Systems Aspects; Services and Service Capabilities (3G TS 22.105 version 3.10.0)".

[12] Web Services Description Language (WSDL), http://www.w3c.org/2002/ws/desc/.

[13] R. Montanari, C. Stefanelli, N. Dulay, "Flexible Security Policies for Mobile Agents Systems", Microprocessors and Microsystems, Elsevier Science, May 2001, pp 93-99.

[14] B. Bhushan, T.Gringel, C. Ryan, et al., "Federated Accounting Management System Architecture for Multimedia Service Usage Management", IFIP/IEEE International Conference on Management of Multimedia Networks and Services (MMNS02), Oct. 2002. 